OPEN ACCESS

Edited by:

Wanping Chen,

Georg-August-University Goettingen,

Germany

Reviewed by:

Yuanliang $\mathrm{Hu}$,

Hubei Normal University, China

Guiming Fu,

Nanchang University, China

*Correspondence:

Bei-Zhong Han

hbz@cau.edu.cn

Specialty section:

This article was submitted to

Food Microbiology,

a section of the journal

Frontiers in Microbiology

Received: 25 May 2021

Accepted: 14 June 2021

Published: 12 July 2021

Citation:

Zhang $Y$, Shen $Y$, Cheng $W$,

Wang $X$, Xue $Y$, Chen $X$ and Han $B-Z$

(2021) Understanding the Shifts

of Microbial Community and Metabolite Profile From Wheat

to Mature Daqu.

Front. Microbiol. 12:714726 doi: 10.3389/fmicb.2021.714726

\section{Understanding the Shifts of Microbial Community and Metabolite Profile From Wheat to Mature Daqu}

\author{
Yuandi Zhang ${ }^{1}$, Yi Shen ${ }^{2}$, Wei Cheng ${ }^{2}$, Xi Wang ${ }^{2}$, Yansong Xue ${ }^{1}$, Xiaoxue Chen ${ }^{1}$ and \\ Bei-Zhong Han ${ }^{1 *}$
}

1 Beijing Laboratory for Food Quality and Safety, College of Food Science and Nutritional Engineering, China Agricultural University, Beijing, China, ${ }^{2}$ Sichuan Langjiu Co., Ltd., Luzhou, China

Wheat-originated microbes play an important role in shaping the quality of hightemperature Daqu which is commonly used as a starter for producing sauce-flavor Baijiu. However, the shifts of microbiota from raw material to fresh Daqu and then to mature Daqu remain unclear. Hence, in the present study, the inner and outer of fresh and mature Daqu were collected to explore the correlation between microbiota and metabolites as well as the source of the microbiota in Daqu. Results indicated that the activities of amylase and protease between the inner and outer of fresh Daqu varied significantly while both parts became similar after maturation. The predominant bacteria shifted from Saccharopolyspora (outer) and Staphylococcus (inner) to Kroppenstedtia (both outer and inner), while the predominant fungi shifted from Thermoascus (both outer and inner) to Byssochlamys (outer) and Fusarium (inner). A combining analysis of headspace solid-phase micro extraction-gas chromatography-mass spectrometry, headspace gas chromatography-ion mobility spectrometry, and nuclear magnetic resonance was employed to detect the metabolites. The network analysis was conducted to perform the relationships between microbes and metabolites. The results showed that the bacteria, especially Saccharopolyspora, Bacillus, and Acinetobacter, had a strong correlation with the productions of esters, amino acids and their derivatives, and sugars and their derivatives, while most fungi such as Thermoascus, were negatively correlated with the phenylalanine, trimethylamine $n$-oxide, and isovalerate. SourceTracker analysis indicated that wheat was the important source of the Daqu microbiota, especially, the microorganisms in the inner of Daqu might be the drivers of the microbial succession during maturation. This study provided a comprehensive exploration to understand the microbial sources and shifts in high-temperature Daqu during maturation.

Keywords: Daqu, maturation, microbiota, metabolites, wheat

\section{INTRODUCTION}

Baijiu is a fermented alcoholic beverage and plays an important role in the history of Chinese food culture for 3000 years (Jin et al., 2017). The popularity of Baijiu to a great extent is due to its rich tastes and characterized flavors, both of which are produced in the complex production processes, including solid-state fermentation, distillation, storage, and blending (Xu et al., 2010). 
Particularly, fermentation plays an important role in the production of volatile flavors and critical secondary metabolites (Zheng et al., 2011). Daqu, made of wheat, barley, pea, and/or corn, is the most common starter and adopted in all the famous Baijiu in China (Wang et al., 2021). Daqu production needs a series of steps, including moistening, breaking, mixing shaping, spreading, incubating, and maturation (Zheng et al., 2011). Among them, maturation is the vital step that involves microbial enrichment, primary and secondary metabolic processes, and changes in diverse microbes and metabolites (Fan et al., 2019).

Daqu production still mainly relies on the experience of the operators to evaluate the quality of the raw materials and the maturation degree of the Daqu, which might not be objective all the time (Deng et al., 2020). The development of the new detection methods, like gas chromatography-mass spectrometry (GC-MS), high performance liquid chromatography-mass spectrometry (HPLC-MS), leads to the discrimination of Daqu maturation easier and more accurate to some extent (Pang et al., 2020). However, the microbiota is the original driver of the maturation. Maturation can promote enriched microbes to interact further, thereby leading to the mixed microbial communities and various metabolites to form balanced and stable Daqu (Gou et al., 2015). Culture-dependent and Illumina MiSeq sequencing methods were used to determine the microbial community during the maturation. A comprehensive understanding of the source and succession of the microbiota and the metabolites is very useful and urgent for the stable production of the high-quality Daqu.

Sauce-flavor Baijiu, one of the most popular Baijiu in China, is famous for its flavor resembling soy sauce, full-body, and longlasting aroma (Zheng and Han, 2016). Its production relies on the high-temperature Daqu and the traditional production steps as shown in Supplementary Figure 1. Recently, microbiological and physicochemical methods were combined to explore the distribution of microbiota and various metabolites in Daqu. Jin et al. (2019) revealed the dominant microbes in Daqu and their distribution characteristics and detected the volatile flavors by headspace solid-phase micro extraction-gas chromatographymass spectrometry (HS-SPME-GC-MS). Their results showed that the dominant bacteria of sauce-flavor Daqu were Bacillales, Enterobacteriales, and Lactobacillales, while the dominant fungi were Trichoderma, Candida, Aspergillus, Thermomyces, and Trichosporon. In Jin et al. (2019) study, the results showed that bacterial diversity was higher than fungal diversity in Daqu, and had the significant differences in microbial composition, distribution and physicochemical indices between the inner and outer parts of Daqu. These studies were all conducted on the mature Daqu and not associated with the changes during the maturation.

In the present study, Illumina MiSeq sequencing was used to investigate the microbiota succession from wheat, to fresh Daqu and mature Daqu. HS-SPME-GC-MS, headspace gas chromatography-ion mobility spectrometry (HS-GC-IMS), and nuclear magnetic resonance $\left({ }^{1} \mathrm{H}\right.$ NMR) were combined to comprehensively evaluate the metabolites in the different parts of Daqu, and different steps of Daqu production. Besides, SourceTracker analysis was employed to evaluate the contribution of wheat to Daqu in microbiota (Jin et al., 2019; Chen et al., 2021). It is the first comprehensive study on shifts in microbiota and metabolites for maturation of the sauce-flavor Daqu and will shed light on the improvement of Daqu production and the quality of the sauce-flavor Baijiu.

\section{MATERIALS AND METHODS}

\section{Sampling}

The samples were collected from a sauce-flavor Baijiu manufacturer in Sichuan, China in 2020. Samples were collected in two different points: fresh Daqu and mature Daqu. Fresh Daqu (F-Daqu) refers to the newly made Daqu that needs 6 months of maturation. Mature Daqu (M-Daqu) refers to Daqu that is ready for Baijiu fermentation. To get reliable samples, Daqu bricks from each step were randomly selected from upper, middle, and lower locations in triplicate. Then each Daqu block was separated into two parts, the surface layer of $2.0 \sim 3.0 \mathrm{~cm}$ thick was named the outer part of Daqu (O-Daqu), and the remaining central part was named the inner part of Daqu (I-Daqu). Raw material (wheat) samples $(3.0 \mathrm{~kg}$ ) were collected randomly from the storage. All samples were made in triplicate. Daqu samples were stored at $4^{\circ} \mathrm{C}$ for microbial counts and $-20^{\circ} \mathrm{C}$ for metabolite analysis and Illumina MiSeq sequencing analysis.

\section{Microbiological Analysis}

Total aerobic bacteria and thermophilic bacteria were enumerated on Plate Count Agar (PCA) (Aobox, Beijing, China), and were cultured at 37 and $55^{\circ} \mathrm{C}$, respectively. Lactic acid bacteria (LAB) were enumerated on LAB culture medium (MRS; Aobox, Beijing, China) with natamycin $500 \mu \mathrm{g} / \mathrm{ml}$. The plates were incubated at $30^{\circ} \mathrm{C}$ for $48-72 \mathrm{~h}$ (Pradhan and Tamang, 2019). Fungi were enumerated on two different media named Malt Extract Agar (MEA) (Aobox, Beijing, China) and Rose Bengal Chloramphenicol Agar (RBCA) (Aobox, Beijing, China), respectively, to which $100 \mathrm{mg} / \mathrm{L}$ chloramphenicol (Oxoid, SR0078E) was added. Colony Forming Units (CFU) were calculated and converted to the value of base 10 Logarithm and recorded as $\log _{10}$ (CFU/g) (Zheng et al., 2012).

\section{Analyses of Physicochemical Properties and Enzymology Properties}

Moisture in Daqu samples was determined with a gravimetric method by drying $5.0 \mathrm{~g}$ samples at $105^{\circ} \mathrm{C}$ for $3 \mathrm{~h}$ (Pang et al., 2020). Daqu powder (5.0 g) was soaked in $50 \mathrm{~mL}$ of distilled water for $30 \mathrm{~min}$, and water extracts were collected after filtration. The $\mathrm{pH}$ was measured using a $\mathrm{pH}$ meter positioned in the slurry (Zheng et al., 2012). The activity of glucoamylase and protease were determined according to the 3,5-dinitrosalicylic acid (DNS) method and Folin-phenol method, respectively (Fan G. S. et al., 2020). One unit of glucoamylase activity was defined as the amount of Daqu required for the liberation of $1 \mu \mathrm{mol}$ glucose per minute in PBS buffer ( $50 \mathrm{mM}, \mathrm{pH} 6.5)$ at $40^{\circ} \mathrm{C}$. One unit of protease activity was defined as the amount of Daqu required for the liberation of $1 \mathrm{mg}$ tyrosine per minute in PBS buffer $(50 \mathrm{mM}$, $\mathrm{pH} 7.0$ ) at $40^{\circ} \mathrm{C}$ (Li et al., 2017). 


\section{Analysis of Volatile Flavors by HS-SPME-GC-MS}

The volatile flavors were analyzed by HS-SPME-GC-MS. The pre-treatment of HS-SPME-GC-MS was carried out as described below: $2.0 \mathrm{~g}$ sample was mixed with $8.0 \mathrm{~mL}$ Milli-Q water and subjected to ultrasonic treatment for 30 min (Liu et al., 2019; Pang et al., 2020). This was carried out in triplicates. Following this, the sample solutions were centrifuged at $6000 \times g$ at $4^{\circ} \mathrm{C}$ for $15 \mathrm{~min}$. Subsequently, $8 \mathrm{~mL}$ supernatant from the samples, and $2.0 \mathrm{~g}$ sodium chloride were placed into a $20 \mathrm{ml}$ vial. This process was repeated with all three (triplicate) samples (Fan Y. et al., 2020). The volatile compounds were extracted with SPME fiber at $50^{\circ} \mathrm{C}$ for $50 \mathrm{~min}$. The contents of volatile compounds were detected by GC-MS (Zhang et al., 2020).

\section{Analysis of Volatile Flavors by HS-GC-IMS}

To investigate the spectrum changes of metabolites in Daqu samples, an untargeted analysis of volatile fingerprints was performed on a GC-IMS system (FlavourSpec ${ }^{\circledR}$, Gesellschaft für Analytische Sensorsysteme $\mathrm{mbH}$, Dortmund, Germany) equipped with an automatic headspace sampler unit (CTCPAL, CTC Analytics AG, Zwingen, Switzerland). Extraction of volatile flavors from Daqu by SPME refers to the method of Yang et al. (2020) with minor modifications. In brief, $2.5 \mathrm{~g}$ of Daqu was weighed and placed into $20 \mathrm{~mL}$ headspace glass sampling vial. Subsequently, samples were incubated at $60^{\circ} \mathrm{C}$ for $15 \mathrm{~min}$. After incubation, $200 \mu \mathrm{L}$ of headspace was automatically injected into the injector. The volatile flavors from the Daqu were analyzed by GC-IMS referring to the method of Li et al. (2019) with minor modifications. The volatile flavors were separated and analyzed using a GC-IMS instrument, equipped with a quartz capillary column (FS-SE54-CB-1, $15 \mathrm{~m} \times 0.53 \mathrm{~mm}, 0.5 \mu \mathrm{m})$. The GC condition is as following: column temperature, $60^{\circ} \mathrm{C}$; carrier gas: ultrapure nitrogen (purity $\geq 99.999 \%$ ); carrier gas flow rate, $2 \mathrm{~mL} / \mathrm{min}$ (0-2 $\mathrm{min}), 2-10 \mathrm{~mL} / \mathrm{min}(2-10 \mathrm{~min}), 10-100 \mathrm{~mL} / \mathrm{min}(10-$ $20 \mathrm{~min}), 100-150 \mathrm{~mL} / \mathrm{min}$ (20-30 min); detection time, $25 \mathrm{~min}$ (Yang et al., 2020). The following conditions were maintained in the automatic headspace sampler condition: incubation temperature, $60^{\circ} \mathrm{C}$; incubation time, $15 \mathrm{~min}$; injection method, headspace injection; injection volume, $200 \mu \mathrm{L}$; injection needle temperature, $85^{\circ} \mathrm{C}$; heating method, shaking heating; shaking speed, $500 \mathrm{r} / \mathrm{min}$; splitless injection (Márquez-Sillero et al., 2011). IMS detection condition is as follows: length of the drift tube, $98 \mathrm{~mm}$; linear voltage in the tube, $500 \mathrm{~V} / \mathrm{cm}$; the temperature of drift tube, $45^{\circ} \mathrm{C}$; drift gas, ultrapure nitrogen (purity $\geq 99.999 \%$ ); the volume of drift gas, $150 \mathrm{~mL} / \mathrm{min}$; radioactive source, $\beta$-rays (tritium, $3 \mathrm{H}$ ); ionization mode, positive ion (Yang et al., 2020).

\section{Analysis of Polar Metabolites by NMR}

The aqueous extracts for NMR measurements were prepared as reported previously. A $600 \mu \mathrm{l}$ aliquot of each sample was transferred into a 5 -mm NMR tube. All ${ }^{1} \mathrm{H}$ NMR spectra were measured at $300 \mathrm{~K}$ using an AVANCE NMR spectrometer (proton frequency 1/4 600.13 MHz, 14.1 T; Bruker, Billerica, Germany) with a cryogenic NMR probe (Wu et al., 2009). The ${ }^{1} \mathrm{H}$ NMR experiments were performed using the following conditions: NOESYGPPRR1D pulse sequence; relaxation delay, $4.00 \mathrm{~s}$; mixing time (for NOESY), $1.00 \mathrm{~s}$; acquisition time, $2.28 \mathrm{~s}$; number of steady states transients (dummy scans), 4; gradient pulse time, $1.00 \mathrm{~ms}$; solvent suppression, pre-saturation with spoil gradient; spectral width, $7184 \mathrm{~Hz}$; and time domain size, $32 \mathrm{k}$ (Li et al., 2018). The compounds were identified and quantified with Chenomx software (version 7.6; Chenomx, Edmonton, Canada) with reference to the internal standard TSP. Each ${ }^{1} \mathrm{H}$ NMR spectrum was equally divided into 242 fragments with width of $0.04 \mathrm{ppm}$. Spectra both with a range of $0.00 \sim 10.00 \mathrm{ppm}$ and exception of residual water resonance $(4.50 \sim 4.80 \mathrm{ppm})$, were divided into $0.04 \mathrm{ppm}$ wide bins, followed by importing the achieved integral values into Microsoft ${ }^{\circledR}$ Excel (Microsoft Corporation, Redmond, WA, United States) (Wu et al., 2009).

\section{DNA Extraction and Illumina MiSeq Sequencing Analysis}

DNA extraction was carried out with E.Z.N.A. ${ }^{\circledR}$ Soil DNA kits (Omega, Norcross, GA, United States) as per the manufacturer's instructions. For bacteria, the V3-V4 domains of the $16 \mathrm{~S}$ rRNA genes were amplified using primers 338F and 806R. For fungi, the internal transcribed spacer (ITS) ITS1 regions were amplified with primers ZIT_F and ZITS_R (He et al., 2019). Reaction conditions consisted of an initial $95^{\circ} \mathrm{C}$ for $3 \mathrm{~min}$ followed by 35 cycles of $95^{\circ} \mathrm{C}$ for $30 \mathrm{~s}, 55^{\circ} \mathrm{C}$ for $30 \mathrm{~s}$, and $72^{\circ} \mathrm{C}$ for $45 \mathrm{~s}$, and a final extension of $72^{\circ} \mathrm{C}$ for $10 \mathrm{~min}$. The purified amplicons were paired-end sequenced on an Illumina MiSeq PE300 system (Illumina, San Diego, CA, United States). Trimmomatic software (version 0.36) was used for mass filtering of the merged sequence (Bolger et al., 2014). Then Uchime software (version 8.1) was used to identify and remove chimeric sequences. Bioinformatics analysis of highquality sequences was performed on a quantitative insight in microbial ecology (QIIME 1.8.0) platform. Clustering operation taxonomic units (OTUs) from clean tags with 97\% similarity was done using the Uparse software (version 9.6). Then the taxonomic information was assigned to all bacterial OTUs via

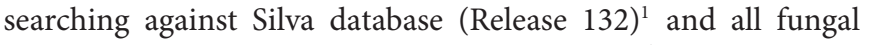
OTUs via searching against Unite (Release 7.2$)^{2}$ by using RDP classifier (version 2.2).

\section{Statistical Analysis}

Statistical analysis was carried out using IBM-SPSS V22.0 (IBM, United States). A one-way ANOVA with Duncan's test was used to determine the significance of physicochemical properties and microbial counts (Li et al., 2020). Principal component analysis (PCA) was performed to analyze the metabolites data by SIMCA-14.1. Paired $t$-test and wilcoxon tests were performed to test the difference in the alpha and beta diversity indices within the stats R package (Version 2.15.3) (Dai et al., 2020).

\footnotetext{
${ }^{1} \mathrm{http} / / /$ www.arb-silva.de

${ }^{2}$ http://unite.ut.ee/index.php
} 
The relationship between microbiota structure and metabolites was analyzed by Two-way Orthogonal Partial Least Squares (O2PLS) and visualized via Cytoscape (v.3.4.0) (Langille et al., 2013). We calculated the Pearson correlation coefficient among the different Daqu samples and raw materials to analyze the relationships among them. SourceTracker (version 0.9.8) was used to analyze the sources of microbial communities in Daqu (Knights et al., 2011).

\section{RESULTS}

\section{Physicochemical Properties}

The physicochemical properties of inner of fresh Daqu (F-I-D), outer of fresh Daqu (F-O-D), inner of mature Daqu (M-I-D), and outer of mature Daqu (M-O-D) were shown in Table 1. The moisture changed with the maturation. Obviously, the moisture in F-Daqu was significantly higher $(P<0.05)$ than $M-D a q u$, and the moisture in I-Daqu was significantly higher $(P<0.05)$ than $\mathrm{O}-\mathrm{Daqu}$. The $\mathrm{pH}$ of I-Daqu decreased slightly while that of ODaqu increased. After the 6 months maturation, both activities of amylase and protease decreased in the I-Daqu, while their activities show a opposite trend.

\section{Microbiological Analysis}

The microbial counts of bacteria and fungi in Daqu samples were shown in Table 2 . The microbial counts in the F-I-D were significantly higher than that in F-O-D $(P<0.05)$ while the microbial counts of M-I-D was close to that of M-O-D. The difference in microbial counts between I-Daqu and O-Daqu reduced during maturation. The microbial counts in I-Daqu decreased while those in O-Daqu increased. The changes of the environment during maturation drove the microbiota inner and outer of Daqu to become similar and eventually to form a stable system.

Illumina MiSeq sequencing was utilized to characterize the microbiota structures in F-I-D, F-O-D, M-I-D, and M-O-D. A total of 983,228 high quality reads from V3-V4 region of $16 \mathrm{~S}$ rRNA gene sequences, and 1,684,859 high quality reads from ITS region were obtained from all samples. For bacteria, there was an average of 49,161 reads per sample, with a range from 29,943 to 73,680 reads. For fungi, there was an average of 84,243 reads per sample, with a range from 32,867 to 144,177 reads. OTUs in samples were defined with

TABLE 1 | Physicochemical properties of Daqu samples.

\begin{tabular}{lccll}
\hline Sample & Moisture (\%) & $\mathbf{p H}$ & $\begin{array}{l}\text { Amylase } \\
\text { Activity (U/g) }\end{array}$ & $\begin{array}{l}\text { Protease } \\
\text { Activity (U/g) }\end{array}$ \\
\hline F-I-D & $17.28 \pm 1.39^{\mathrm{d}}$ & $6.38 \pm 0.05^{\mathrm{c}}$ & $7.40 \pm 0.14^{\mathrm{d}}$ & $14.80 \pm 2.18^{\mathrm{b}}$ \\
F-O-D & $12.60 \pm 0.35^{\mathrm{c}}$ & $4.74 \pm 0.13^{\mathrm{a}}$ & $0.73 \pm 0.02^{\mathrm{a}}$ & $1.28 \pm 0.34^{\mathrm{a}}$ \\
M-I-D & $9.24 \pm 0.26^{\mathrm{b}}$ & $6.29 \pm 0.07^{\mathrm{C}}$ & $4.35 \pm 0.03^{\mathrm{C}}$ & $12.67 \pm 1.59^{\mathrm{b}}$ \\
M-O-D & $7.63 \pm 0.27^{\mathrm{a}}$ & $5.77 \pm 0.08^{\mathrm{b}}$ & $2.16 \pm 0.03^{\mathrm{b}}$ & $2.87 \pm 1.59^{\mathrm{a}}$ \\
\hline
\end{tabular}

Values represent means $\pm S D(n=3)$.

Means with different superscripts are significantly different (One-Way ANOVA; $P<0.05)$.
TABLE 2 | Microbial counts in Daqu samples.

\begin{tabular}{lcccc}
\hline Log $_{10}$ CFU/g & \multicolumn{2}{c}{ F-Daqu } & \multicolumn{2}{c}{ M-Daqu } \\
\hline $\begin{array}{l}\text { Microbial } \\
\text { groups }\end{array}$ & F-I-D & F-O-D & M-I-D & M-O-D \\
$\begin{array}{l}\text { Mesophilic } \\
\text { aerobic } \\
\text { bacteria }\end{array}$ & $8.36 \pm 0.11^{d}$ & $4.73 \pm 0.07^{a}$ & $5.64 \pm 0.07^{c}$ & $5.00 \pm 0.04^{b}$ \\
$\begin{array}{l}\text { Thermophilic } \\
\text { bacteria }\end{array}$ & $6.96 \pm 0.07^{d}$ & $4.63 \pm 0.11^{a}$ & $5.73 \pm 0.13^{c}$ & $5.10 \pm 0.06^{b}$ \\
Lactic acid & $8.28 \pm 0.02^{d}$ & $3.64 \pm 0.07^{a}$ & $4.94 \pm 0.03^{b}$ & $5.06 \pm 0.07^{c}$ \\
bacteria & & & & \\
$\begin{array}{l}\text { Fungi on RBCA } \\
\text { Fungi on MEA }\end{array}$ & $8.22 \pm 0.04^{d}$ & $2.48 \pm 0.01^{a}$ & $5.17 \pm 0.02^{c}$ & $4.47 \pm 0.04^{b}$ \\
& $8.21 \pm 0.02^{d}$ & $2.49 \pm 0.01^{a}$ & $5.29 \pm 0.03^{c}$ & $4.68 \pm 0.03^{b}$
\end{tabular}

Values represent means $\pm S D(n=3)$.

F-I-D: Inner of fresh Daqu; F-O-D: Outer of fresh Daqu; M-I-D: Inner of mature Daqu; M-O-D: Outer of mature Daqu.

Cluster analysis was based on Unweighted UniFrac distance matrix and the UPGMA method. Means with different superscripts are significantly different (One-Way ANOVA; $P<0.05)$.

a $\geq 97 \%$ sequence identity cutoff, and all samples had high Good's coverage (1.00). Chaol richness and other information were shown in Supplementary Table 1. The rarefaction curves of both bacterial and fungal communities approached the saturation plateau, which indicated that the microbial communities were well represented at the sequencing depth (Supplementary Figure 2).

Taxonomic classification of sequences from bacterial communities (a) and fungal communities (b) was showed in Supplementary Figure 3. As for bacterial communities, several types of microbial abundance of Daqu such as Lactobacillus, Sphingobacterium, and Staphylococcus decreased after maturation while the abundance of Kroppenstedtia and Lentibacillus increased, indicating that maturation-related changes had occurred at the genus level (Supplementary Figure 3A). As for fungal communities, the results showed that Thermoascus, as the absolute dominant flora, significantly decreased in abundance during the maturation (Supplementary Figure 3B).

The distribution of microbiota at the genus level was shown in Figure 1. For bacterial communities, Staphylococcus predominated in the F-I-D with an average abundance of $70.14 \%$, followed by Kroppenstedtia (24.46\%). After maturation, Kroppenstedtia $(84.88 \%)$ became the predominant bacterial genus, followed by Staphylococcus (1.68\%). Besides, Saccharopolyspora and Kroppenstedtia predominated at the F-O-D with an average abundance of 35.71 and $31.58 \%$, respectively. In M-O-D, Kroppenstedtia (82.60\%) became the predominant bacterial genus, followed by Saccharopolyspora (3.40\%). After maturation, the bacterial compositions in both M-I-D and M-O-D became similar (Figure 1A). As for fungal communities (Figure 1B), Thermoascus possessed an absolutely dominant position in both F-I-D and F-O-D. After maturation, the proportion of Fusarium was increased both in the M-I-D and M-O-D from the fresh Daqu. The relative abundance of Byssochlamys in M-O-D increased significantly (from 0.27 to $24.46 \%$ ). Besides, it was mainly the rise of Microascus 

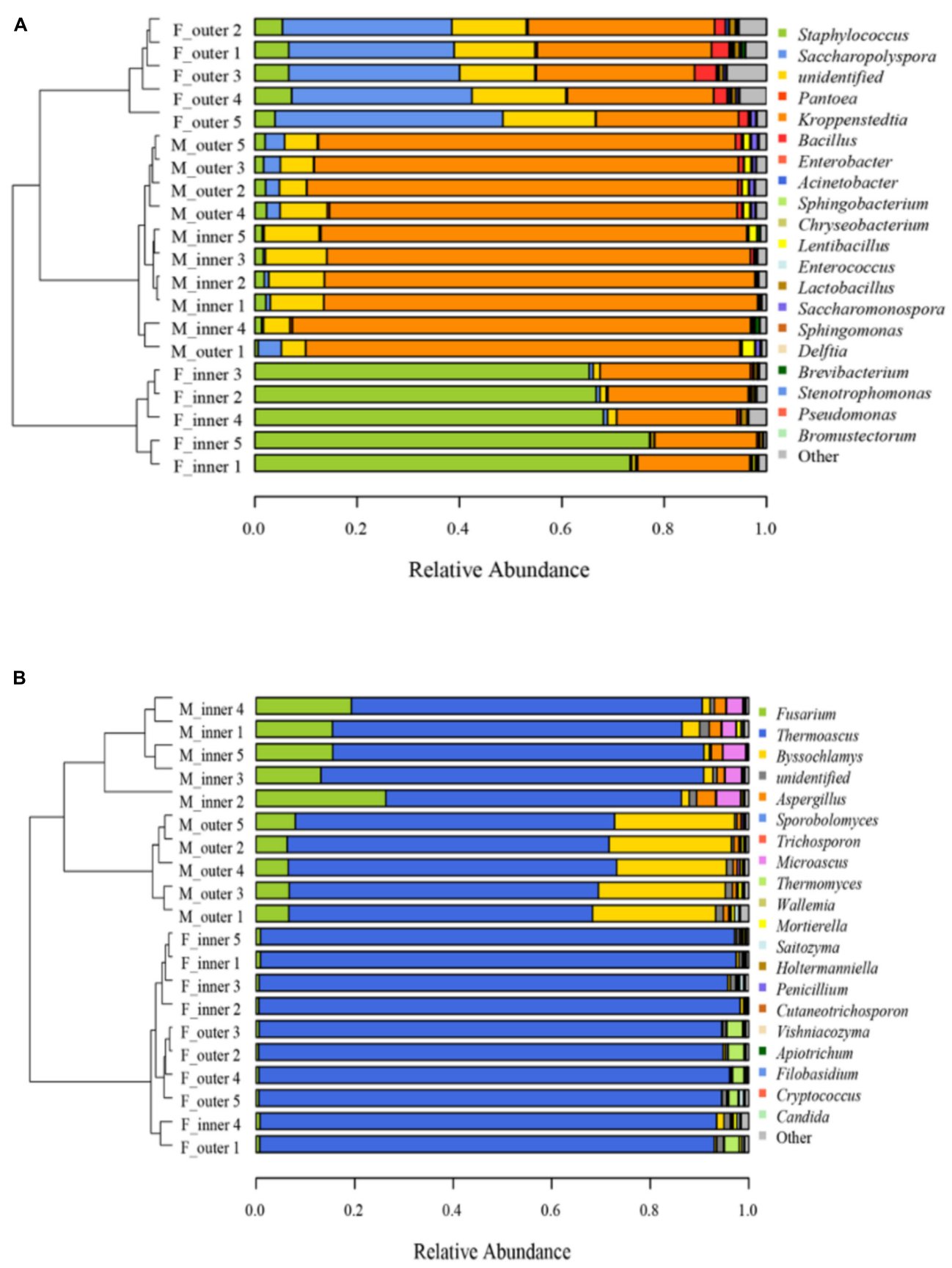

FIGURE 1 | Microbial communities of Daqu samples (A) Bacterial community. (B) Fungal community. F-inner: Inner of fresh Daqu; F-outer: Outer of fresh Daqu; M-inner: Inner of mature Daqu; M-outer: Outer of mature Daqu. Cluster analysis was based on Unweighted UniFrac distance matrix and the UPGMA method. Genus level $(n=5)$.

and Aspergillus (from 0.03 to $3.80 \%$ and from 0.24 to $2.48 \%$, respectively) in M-I-D.

\section{Multivariate Analysis of Volatile Flavors of Daqu}

A total of 76 volatile flavors were detected from both F-Daqu and M-Daqu by HS-SPME-GC-MS, including 11 esters,
15 alcohols, 2 organic acids, 11 ketones, 8 aldehydes, 6 phenols, 5 pyrazine, and 18 others (Supplementary Table 2). Partial least squares discriminant analysis (PLS-DA) was used to analyze the volatile flavor, and the substances with lower variable importance in the projection (VIP) value were eliminated. As shown in Figure 2, the results explained $47.5 \%$ of the total variance with R2X (30.7\%) and R2Y (16.8\%). 


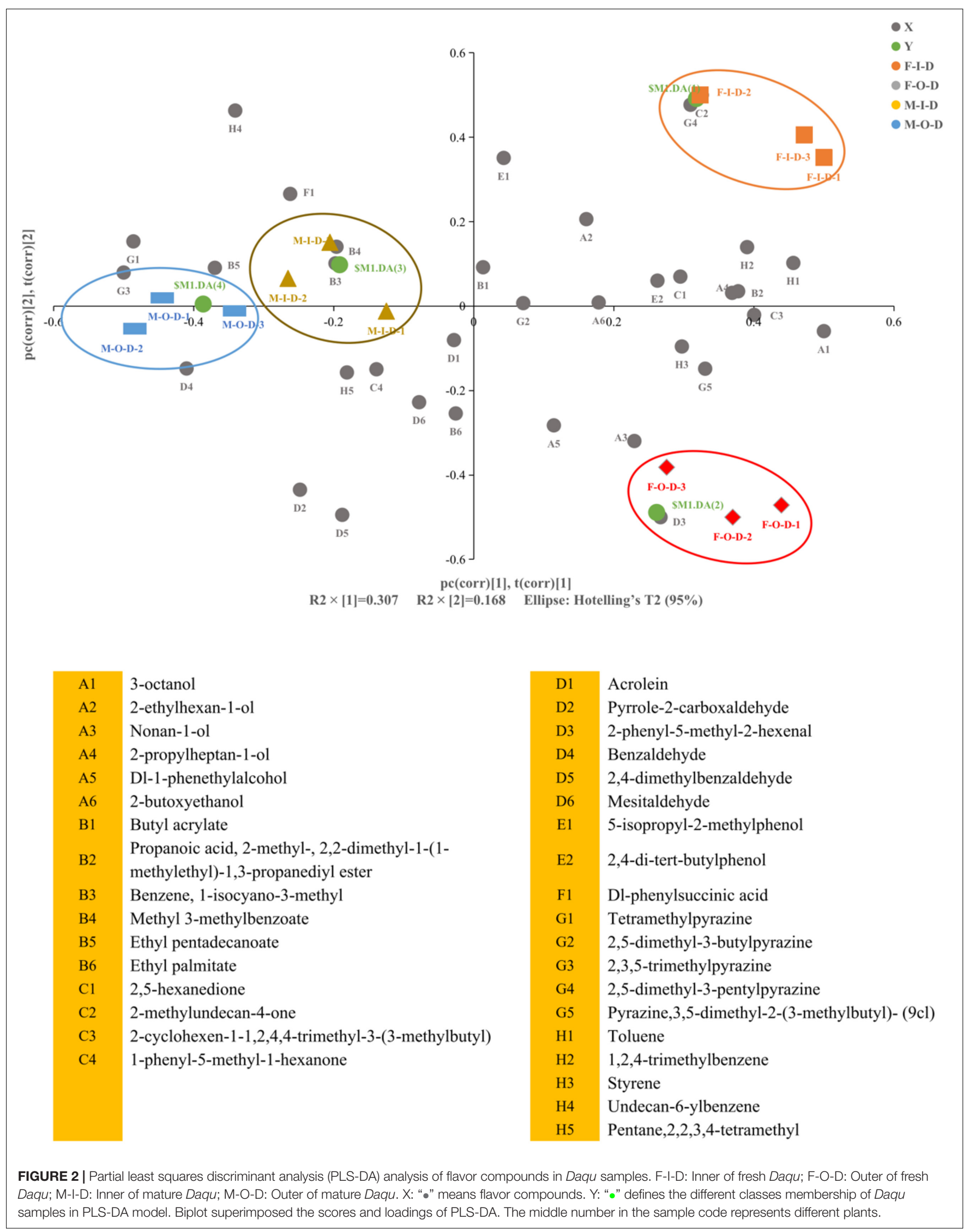




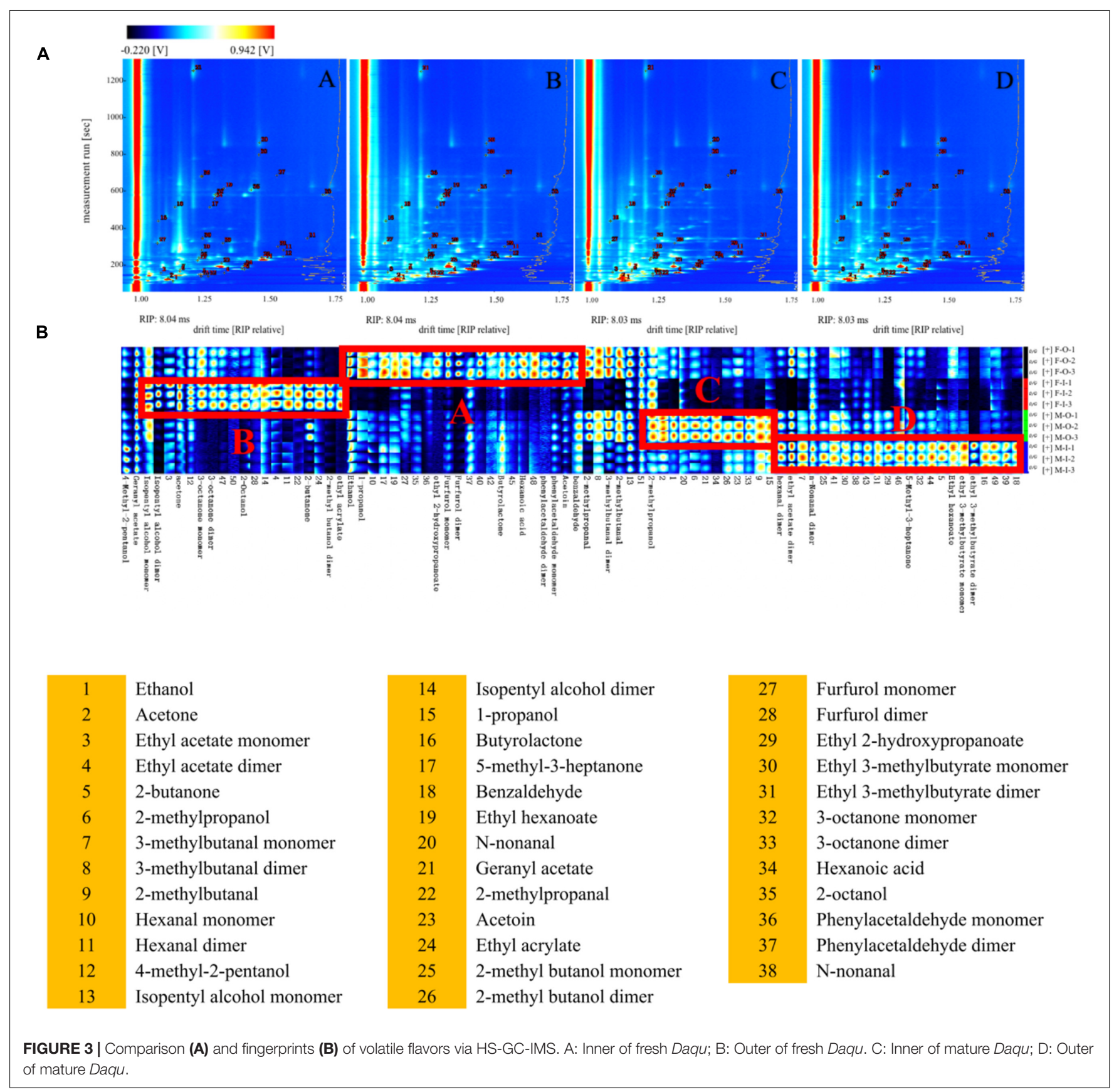

The detailed distinctions of volatile flavors profiles in different samples were revealed by the loading plot of PLS-DA. Terms with large VIP were the most relevant for explaining Y. The VIP of benzene, 1-isocyano-3-methyl, 2-phenyl-5-methyl-2hexenal, 2,4-dimethylbenzaldehyde, 2-methylundecan-4-one, undecan-6-ylbenzene, pyrrole-2-carboxaldehy, 2,5-dimethyl3-pentylpyrazine, mesitaldehyde, ethyl pentadecanoate, 5-isopropyl-2-methylphenol, dl-1-phenethylalcohol, 2,3,5trimethylpyrazine, tetramethylpyrazine, toluene, and 3-octanol (VIP > 1.0) contributed to the specificity of Daqu samples. Detailed VIP value was provided in the Supplementary Table 3. The categories and the content of volatile flavors decreased after maturation. The main differences between F-I-D and F-O-D were shown in the categories and the content of aldehydes and organic acids (Supplementary Table 2). After maturation, the total number of volatile flavors categories of I-Daqu had little change. However, the categories of alcohols, esters and aldehydes increased, while the types of aldehydes and alkanes decreased. It is worth noting that the direct contact between the O-Daqu and air resulting in the volatization of some volatile flavors and the decreased abundance of flavor-producing microorganisms (e.g., Saccharopolyspora) in O-Daqu during the maturation, contributing to the sharp decrease in categories of volatile flavors in O-Daqu (Yang et al., 2020). 
As a new flavor detection technology, GC-IMS is widely used for analyzing volatile flavors under the normal atmospheric pressure and highly sensitive to compounds with high electronegativity, which can be used as a supplementary tool for GC-MS to conduct a more comprehensive and systematic detection of the volatile flavors of Daqu. The two-dimensional imaging of GC-IMS consisted of drift time, retention time, and the intensity of the ion signals. A total of 88 peaks were detected in Daqu samples, and 37 compounds, including 14 aldehydes, 7 alcohols, 6 ketones, 1 organic acid, and 9 esters, were identified by the NIST 2014 and IMS database (Supplementary Table 4). Many volatile flavors present in different degree of polymerization, such as monomers and dimers depending upon their concentrations. Figure $\mathbf{3 A}$ displayed that these products pass through the drift region and multiple signals can be observed as a single compound due to the formation of adducts between the ions and neutral molecules (such as dimers and trimers), including 2-methyl butanol, 2-methylbutanal, 3-methylbutanal, 3-octanone, ethyl 2-hydroxypropanoate, ethyl acrylate, furfurol dimer, hexanal, isopentyl alcohol, n-non-anal, and phenylacetaldehyde. These products exhibited similar retention times, but different drift time (Fan et al., 2021). Figure 3B displays that all compounds, identified via GC-IMS, have been selected to compare the differences of four types of Daqu samples. Each column represented the signal peak of one volatile compound. The brighter spot indicated the higher concentration of the volatile compound. The monomers and dimers of the same compound were indicated by different columns with the same compound name. However, due to the proton affinity and higher concentration, the drift time of dimers increased and showed distinct spots in the fingerprint (Yang et al., 2020). The total volatile flavors of F-O-D and M-O-D were relatively similar, and the same condition was shown in M-O-D and M-I-D based on the spot color. Compounds of regional A in Figure 3B (ethanol, ethyl 2-hydroxy propyl alcohol, propionic acid, furfural, butyrolactone, caproic acid, benzene acetaldehyde, ethyl benzoin, benzene, and formaldehyde, etc.), regional B (isoamyl alcohol, acetone, ketone, octanol, butanone, 2-methyl ethyl acrylate, and butyl alcohol), regional C (2-methyl propyl alcohol, etc.), and regional D (hexanal, ethyl acetate, nonyl aldehyde and 3-methyl ethyl caproate, ethyl butyrate, etc.) represented the characteristics of volatile flavors in F-O-D, F-I-D, M-O-D, and M-I-D.

\section{Polar Metabolites of Daqu}

Nuclear magnetic resonance $\left({ }^{1} \mathrm{H}\right.$ NMR spectroscopy) coupled with multivariate statistical analysis was used to investigate the differences of the Daqu samples in the polar metabolic changes to better characterize Daqu maturation. As shown in Supplementary Table 5, a total of 76 polar metabolites including 17 esters, 19 amino acids and their derivatives, 6 alkalamides, 4 alcohols, 30 sugars and their derivatives, and salt of organic acid were detected in Daqu samples. Due to a series of complicated metabolism like the Maillard reaction and enzymatic reaction, the polar metabolites in Daqu increased with the maturation. For example, in terms of the type and content of esters, F-I-D and F-O-D differed greatly, while M-I-D and M-O-D became closer at the end of maturation. These trends were caused by the shifts of microbiota during maturation. Of all the polar metabolites, the most abundant compounds were alcohols, especially ethanol and glycerol. They were the substrates or precursors for a series of subsequent reactions (Zheng et al., 2011). The polar metabolites' contents especially esters, amino acids and sugars in F-I-D were significantly lower than those in F-O-D.

\section{Network Analysis of the Interactions Among Microbiota, Polar Metabolites and Volatile Flavors in Daqu}

We analyzed the correlations among microbiota, polar metabolites, and volatile flavors in Daqu via Pearson correlation analysis (Figure 4) and the detailed value of Pearson correlation coefficients (Pearson correlation $>|0.7|$ ) was shown in Supplementary Table 6. Correlation analysis between microbiota and polar metabolites was shown in Figure 4A. Bacteria such as Saccharopolyspora, Bacillus, and Acinetobacter showed a strong correlation with polar metabolites. Saccharopolyspora had the highest abundance in F-O-D, which produced more polar metabolites such as esters, amino acids and their derivatives, and sugars and their derivatives in the production process of Daqu. For fungi, the correlation network showed that Thermoascus was negatively correlated with phenylalanine, trimethylamine n-oxide, and isovalerate. In previous studies, Thermoascus could produce some thermostable enzyme and epipolythiodioxopiperazine metabolite derived from the amino acid pathway, and these metabolites exhibited antibacterial and antiviral properties (Leite et al., 2008; Siddiquee, 2018). The abundances of Fusarium, Byssochlamys, Aspergillus, and Microascus in M-Daqu were significantly higher than those in F-Daqu and they showed a strong correlation with 23 polar metabolites including amino acids and their derivatives and esters such as 3-hydroxyisovalerate, propionate, fumarate, acetoacetate, and methionine. These fungi were closely related to the synthesis of most esters and some amino acids during maturation.

Correlation analysis between microbiota and volatile flavors showed that the abundance of Kroppenstedtia was positively correlated with tetramethylpyrazine, 2,3,5-trimethyl pyrazine, 2,4-dimethyl benzaldehyde, hexanal monomer, and ethyl pentadecanoate (Figure 5B). The abundance of Kroppenstedtia increased significantly after Daqu maturation, and the content of associated pyrazines such as tetramethylpyrazine and 2,3,5trimethyl pyrazine were also significantly higher in mature Daqu than those in fresh Daqu. Interestingly, Kroppenstedtia's abundance was negatively correlated with 3-octanone and 3-octanol, while Thermoascus's abundance was positively correlated with these substances. From the point of view of the microbiota succession, Kroppenstedtia and Thermoascus showed the rule of ebb and flow because Kroppenstedtia had a higher tolerance to ethanol which was accumulated in the later step of Daqu maturation. Bacillus has a positive correlation with esters, sugars and derivatives, organic acids, aldehydes and ketones due to its high-temperature resistance and strong secretion enzyme function (Siddiquee, 2018). Fusarium and Aspergillus 


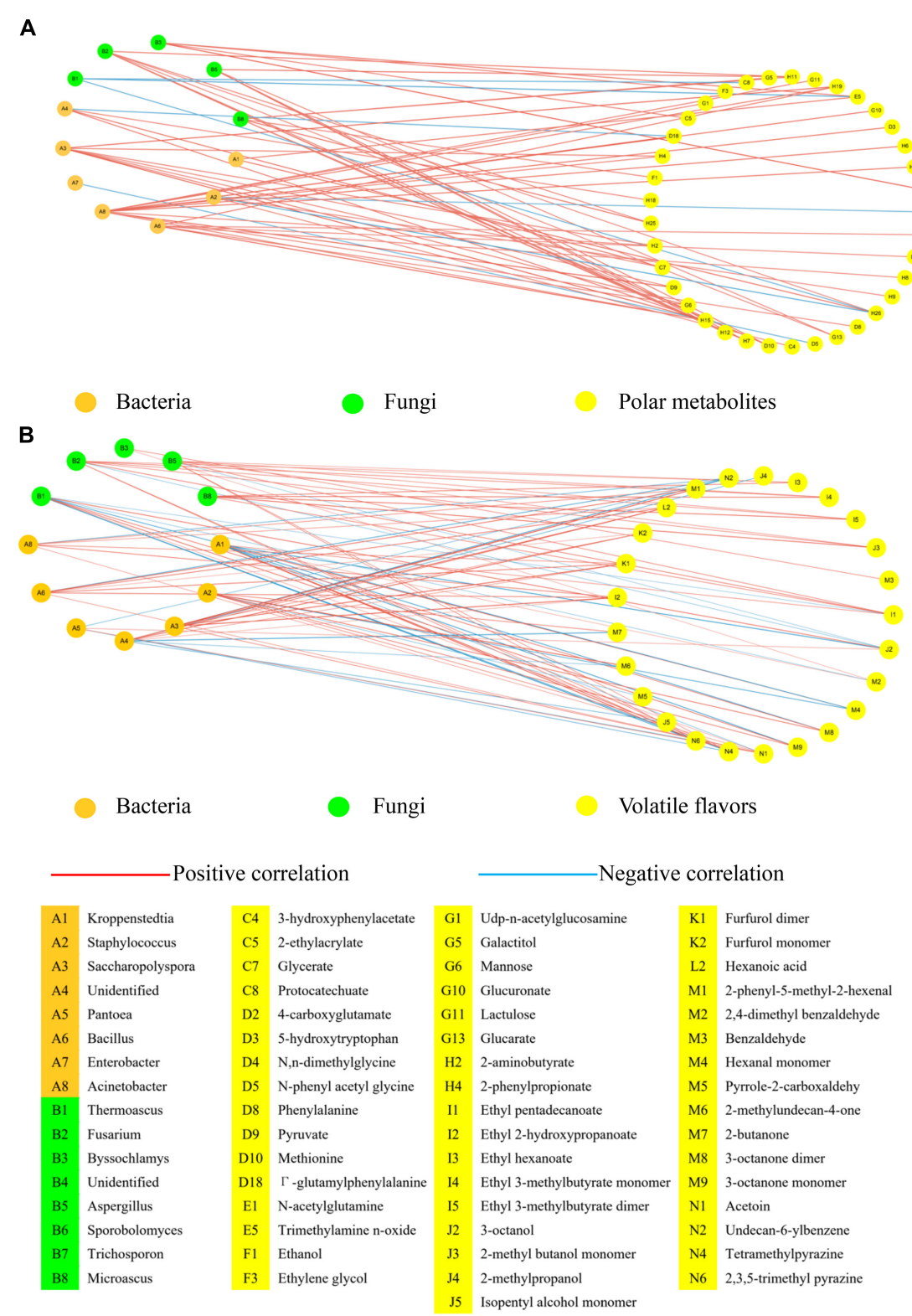

FIGURE 4 | Network analysis of the interactions among microbiota, polar metabolites ( ${ }^{1} \mathrm{H}$ NMR) and volatile flavors (HS-SPME-GC-MS and HS-GC-IMS). (A) The correlated network between microbiota and polar metabolites. (B) The correlated network between microbiota and volatile flavors. Population data was considered at the OTU level and statistically significant Pearson correlations were calculated among Daqu samples. A connection stands for a significant $(P<0.05)$ and positive (Pearson correlation $>|0.7|)$ correlation.

had a similar correlation with volatile flavors, and both had a strong positive correlation with esters and alcohols. Studies have pointed out that Fusarium could decompose cellulose and other organic compounds and could degrade phenolic compounds and polycyclic aromatic hydrocarbons at the same time (Leite et al., 2008). Aspergillus could decompose proteins to produce volatile flavors and also produce organic acids (Yu et al., 2021).

Kroppenstedtia was positively correlated with some pyrazines such as tetramethylpyrazine and 2,3,5-trimethyl pyrazine. Other bacteria such as Saccharopolyspora, Bacillus, and Acinetobacter were positively correlated with organic acid, amino acids and their derivatives, and sugars and their derivatives. Besides, Fusarium and Aspergillus had a strong positive correlation with esters, alcohols, organic acid while Thermoascus showed a negative correlation with phenylalanine, trimethylamine n-oxide, and isovalerate.

\section{SourceTracker Analysis of Raw Materials and Daqu}

SourceTracker has been widely used as an important tool of tracking microbial source in many fields, including food 

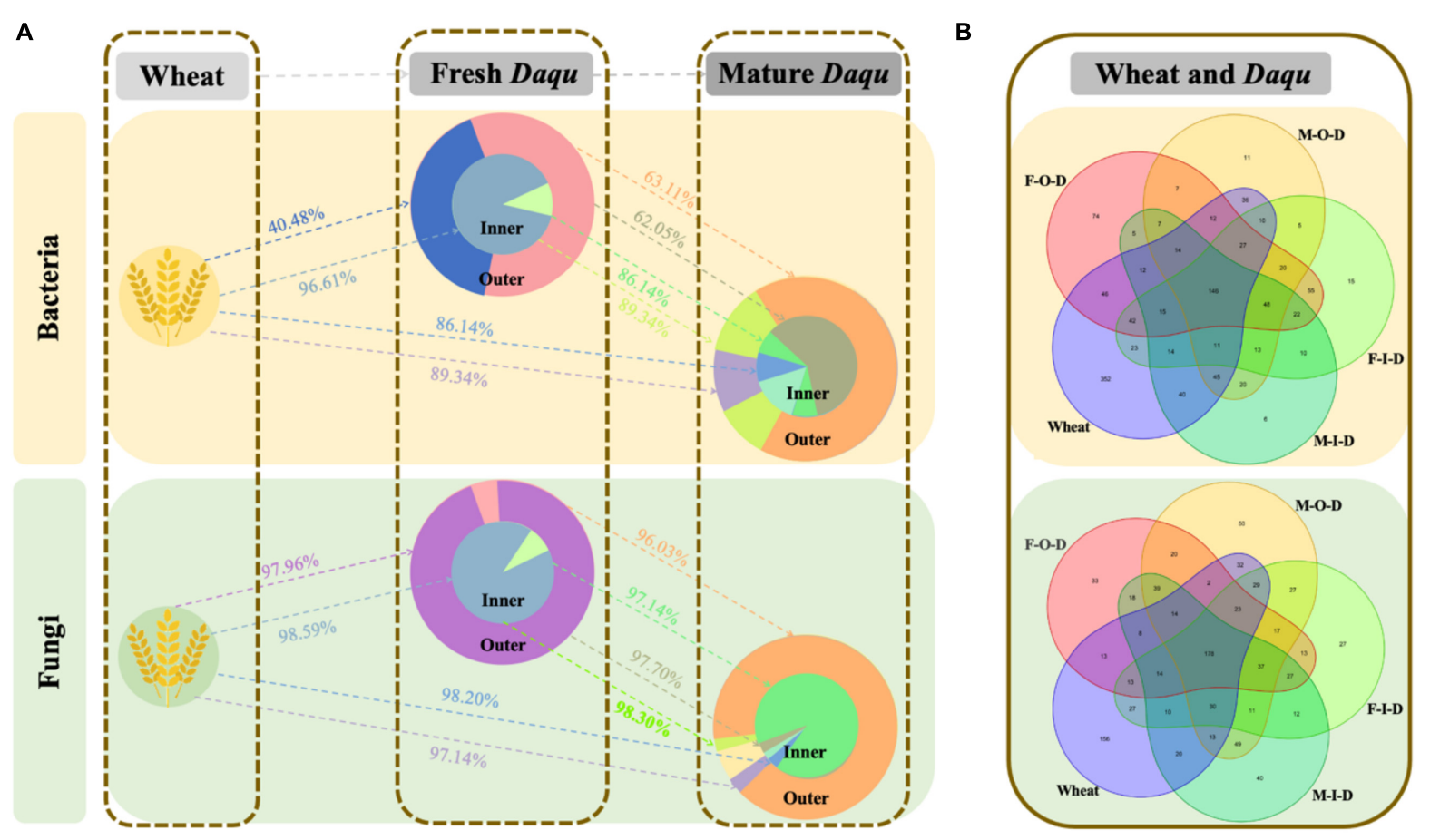

FIGURE 5 | SourceTracker results for the wheat and Daqu sample via SourceTracker. (A) Estimation of shift pathway and proportion. (B) Specific numbers of OTUs. The " $\rightarrow$ " represent microbiota shift direction. F-I-D: Inner of fresh Daqu; F-O-D: Outer of fresh Daqu; M-I-D: Inner of mature Daqu; M-O-D: Outer of mature Daqu.

fermentation and meat processing (Du et al., 2019). Thus, we exploited SourceTracker to estimate the source and succession of microbiota in Daqu. In addition to the microbiota in Daqu, we also analyzed the microbiota on the surface of wheat, the raw material for Daqu-making (Supplementary Table 7). The traceability of microbiota in high-temperature Daqu were shown in Figure 5. Schematic diagram showed that the microbiota shifted from wheat to fresh Daqu and finally to mature Daqu. In particular, the proportion of microbiota shifted as shown in Figure 5A and the specific number of OTUs were shown in Figure 5B. The bacterial community of F-I-D mainly came from wheat $(96.61 \%)$, while only about $40.48 \%$ of F-O-D came from wheat. In detail, Staphylococcus, Saccharopolyspora, and Kroppenstedtia might shifted to Daqu from wheat and became dominant bacteria in Daqu. Besides, most of the wheat-based microbiota were shown in the I-Daqu (Du et al., 2019). Compared with F-Daqu, M-Daqu were more from the F-I-D rather than the F-O-D. Approximately $89.34 \%$ of bacterial community in M-O-D was shifted from F-I-D, slightly higher than that in M-I-D (86.14).

Combined with the results of Illumina MiSeq sequencing, the fungal traceability of I-Duqu and O-Daqu was similar. However, the difference of fungal community (Figure 1B) in M-I$\mathrm{D}$ and $\mathrm{M}-\mathrm{O}-\mathrm{D}$ after maturation became larger than that of the maturation at the beginning. Thermoascus, the dominant fungus with the highest abundance in the Daqu maturation, was also detected on the surface of wheat. It could be inferred that part of Thermoascus shifted from wheat to the F-Daqu and then its abundance reduced due to the growth of thermophilic bacteria and Byssochlamys (Du et al., 2019). There were also some fungi such as Fusarium and Aspergillus in wheat and M-Daqu with high abundance, but low abundance in F-Daqu.

\section{DISCUSSION}

This study explored the microbiota from wheat and the contribution of the inner and outer of Daqu microbiota during maturation. Physicochemical properties, including moisture, $\mathrm{pH}$, amylase activity, and protease activity reflect the maturity and quality of Daqu (Deng et al., 2020). The moisture in F-Daqu was higher than that in M-Daqu, and the moisture in I-Daqu was higher than that of O-Daqu. It is due to the open and wellventilated storage condition and the dry and cool air in the environment, which lead more moisture loss in O-Daqu (Chen et al., 2021). The difference in $\mathrm{pH}$ is related to the difference in composition of organic acids and the changes in the diversity of slightly acidic bacteria like the Acetobacter and Lactobacillus (Fan et al., 2019). The changes in the microbiota that produced amylase and protease might be the mainly reason for the changes in enzyme activities (Guan et al., 2020).

During the maturation, the microbiota in different parts of Daqu underwent great changes. The bacterial communities of F-I-D and F-O-D were quite different because microbes from the environment had a great influence on F-O-D exposed to the air during the whole production process, while most of the microbiome in F-I-D came from raw materials ( $\mathrm{He}$ et al., 2019). Besides, the bacterial community of M-I-D and M-O-D became similar after maturation. Compared 
with medium-temperature and low-temperature Daqu, hightemperature Daqu had relatively fewer bacterial species due to high fermentation temperature (Zheng et al., 2011). The abundance of Saccharopolyspora was high in F-O-D, and the microbiota came from the environment or the raw materials that provided the initial microbiota for enzymatic reactions to produce metabolites. In the mature step of Daqu, the microbial abundance in Daqu decreased after high temperature fermentation, mainly including some thermotolerant and thermophilic bacteria. Kroppenstedtia became the absolute dominant strain in the M-Daqu. It was isolated recently and was identified in several types of Daqu (Fan et al., 2019). However, its role in Daqu manufacture is not clear. Studies had shown that Lactobacillus could inhibit Kroppenstedtia to a certain extent, which explained that the decline of Lactobacillus abundance promoted the growth of Kroppenstedtia after maturation (Yan et al., 2016). For Staphylococcus, the absolute dominant bacterial genera in F-I-D, Stevens et al. (2015) discovered that Staphylococcus were dominant across different foot sites and comprised almost the entire bacterial population on the plantar surface (Stevens et al., 2015). Du et al. (2019) proved that both the raw materials and the environments acted as important sources for Daqu microbiota (Du et al., 2019). Because of the relatively open processing environment and the barefoot stepping process by women, there might be some microbiota such as Staphylococcus originating from the environment or women' feet in Daqu (Wang et al., 2021). During maturation, it was gradually inhibited by Kroppenstedtia (He et al., 2019). For fungal communities, few fungi can stand the temperature in the high-temperature Daqu making process because they prefer the low temperature environment for reproduction. However, the microflora showed a tendency of enrichment after maturation. Specifically, the abundance of Fusarium, Byssochlamys, Aspergillus, and Microascus in mature Daqu was significantly higher than that in fresh Daqu. Fusarium was widely found in nature especially in crops such as wheat and is beneficial to agriculture such as gibberellin (Franco et al., 2021). Fusarium in fresh Daqu was mainly derived from wheat and had a certain inhibitory effect on Thermoascus in the maturation process and became one of the dominant strains in mature Daqu (He et al., 2019). As the absolute dominant fungus in fresh Daqu, Thermoascus was negatively correlated with ethanol, and the accumulation of ethanol in the maturation process had a certain inhibitory effect on it (Wei et al., 2021).

The maturation of Daqu involves the changes of temperature, and the competition between bacteria and fungi lead to the change of volatile flavors produced by their metabolisms, which is also the reason for the production of the abundant volatile flavors in Daqu (Chen et al., 2021). From all volatile flavors detected by HS-SPME-GC-MS and HS-GC-IMS, there were obvious differences among the four Daqu samples. The incubation temperature of sauce-flavor Daqu is usually very high (reaching up to $70^{\circ} \mathrm{C}$ ), and the Maillard reaction causes color changes and produces a series of volatile flavors like ketones, aldehydes, and heterocyclic compounds (Zheng et al., 2011). Besides, Sauceflavor Daqu bricks are big and heavy (above $4.8 \mathrm{~kg}$ ), which results in the different temperatures of the I-Daqu and ODaqu and color gradients from brown inside to yellow outside (Huang et al., 2020). According to the detected results, F-I-D and F-O-D show great differences in compound compositions because of the metabolic reactions at different temperatures before maturation. At the end of maturation, the compositions of M-I-D and M-O-D became similar. It is probably because microbial succession, and compounds exchange took place in the inner and outer Daqu, leading to the formation of a relatively uniform system during maturation. Longitudinally, microbiota succession had been in progress in the whole maturation period. The bacterial community structures of F-Daqu and MDaqu were very different, mainly reflected in the abundance of Kroppenstedtia, Saccharopolyspora, Staphylococcus, and Bacillus. High-intensity Staphylococcus produced volatile flavors such as 3-methyl-1-butanol, 2-butanone, and acetoin, which might play important roles in Baijiu production (Guan et al., 2020). Bacillus could convert starch and proteins into glucose and amino acids by secreting amylase, protease, cellulases, glucanases, and other enzymes, thereby contributing to the development of volatile flavors (Fan G. S. et al., 2020). At the same time, F-Daqu and M-Daqu showed significant differences in fungal community structure. After maturation, the abundance of Fusarium and Byssochlamys increased significantly, and the interaction between microorganisms became more complex. This played a decisive role in the change of metabolites between F-Daqu and MDaqu. Besides, ${ }^{1} \mathrm{H}$ NMR spectroscopy was used to investigate the differences of the Daqu samples in the polar metabolic changes. The results show that esters, amino acids and sugars in F-I-D were significantly lower than those in F-O-D. This was probably because the abundance of Thermomyces in F-O$\mathrm{D}$ was significantly higher than that in F-I-D. Thermomyces secreted cellulase, amylase, and protease, which converted starch, cellulose, and other raw substances into small molecules such as glucose and amino acids. These small molecules supplied nutrition and energy for the growth and metabolism of the microbial community (Ali et al., 2019). The contents of esters in $\mathrm{M}-\mathrm{I}-\mathrm{D}$ and $\mathrm{M}-\mathrm{O}-\mathrm{D}$ were similar because of the similar ester producing bacterial community structure between M-I-D and M-O-D, while the higher abundance of Aspergillus in M-I$\mathrm{D}$ contributed to the significantly higher contents of amino acids and derivatives, sugars and their derivatives in M-I-D than M-O-D due to Aspergillus's ability to decompose the large molecules such as starch into small polar metabolites (e.g., glucose) (Ali et al., 2019).

Some studies in medium temperature Daqu had shown that fungal communities were mainly originated from Daqu making environments (especially tools and indoor ground) while most of bacterial communities were from raw materials ( $\mathrm{Du}$ et al., 2019). During the maturation, the bacterial community of wheat participated in microbiota succession and showed a migration phenomenon from inner to outer of Daqu. In other words, IDaqu was an important channel for wheat microorganisms to act on Daqu (from wheat to F-I-D to the whole Daqu). Some bacteria migrated to the O-Daqu from I-Daqu, mainly Kroppenstedtia, Saccharopolyspora, and Staphylococcus, etc., which resulted in a similar and stable community structure in M-I-D and M-O-D. 
In short, the core bacterial community in the F-I-D was the most important driving force for the maturation of Daqu. Wheat might be the source of these fungi whose growth was inhibited during the Daqu making (Yu et al., 2021). Another important reason was that these fungi in the environment transferred to the outer of Daqu and enriched in the maturation process and reproduced during maturation under suitable moisture and temperature, and finally increased its abundance in mature Daqu (Du et al., 2019). On this basis, the mechanism of raw material microbiota in Daqu still needs to be further studied.

\section{CONCLUSION}

Taken together, wheat-originated microbes were an important source for the microbial community formation of Daqu, especially the inner of Daqu. The bacterial community in the inner of Daqu was an important driving force of maturation. During the maturation, the difference in the bacterial community between the inner and outer of Daqu decreased and the predominant microbes shifted from Saccharopolyspora (outer) and Staphylococcus (inner) of fresh Daqu to Kroppenstedtia (both outer and inner) of mature Daqu, in which was indicated by the difference in metabolites. For fungal community, the predominant fungi shifted from Thermoascus (both outer and inner) in fresh Daqu to Byssochlamys (outer) and Fusarium (inner) in mature Daqu. Bacteria such as Saccharopolyspora, Bacillus, and Acinetobacter had a strong correlation with the contents of esters, amino acids and their derivatives, and sugars and their derivatives, which confirmed the important role of bacterial community in the inner of Daqu. Our study provided a deeper understanding of the role of microbiota during Daqu maturation and improvement of the quality and stability of Daqu.

\section{REFERENCES}

Ali, B., Yi, Z. L., Fang, Y., Chen, L. C., He, K. Z., Liu, D. Y., et al. (2019). Characterization of a fungal thermostable endoglucanase from Chinese Nongflavor Daqu by metatranscriptomic method. Int. J. Biol. Macromol. 121, 183190. doi: 10.1016/j.ijbiomac.2018.09.173

Bolger, A. M., Lohse, M., and Usadel, B. (2014). Trimmomatic: a flexible trimmer for Illumina sequence data. Bioinformatics 30, 2114-2120. doi: 10 . 1093/bioinformatics/btu170

Chen, Y. R., Li, K. M., Liu, T., Li, R. Y., Fu, G. M., Wan, Y., et al. (2021). Analysis of difference in microbial community and physicochemical indices between surface and central parts of Chinese special-flavor Baijiu Daqu. Front. Microbiol. 11:1-12. doi: 10.3389/fmicb.2020.592421

Dai, Y. J., Tian, Z. G., Meng, W. N., and Li, Z. J. (2020). Microbial diversity and physicochemical characteristics of the maotai-flavored liquor fermentation process. J. Nanosci. Nanotechnol. 20, 4097-4109. doi: 10.1166/jnn.2020. 17522

Deng, L., Mao, X., Liu, D., Ning, X. Q., Shen, Y., Chen, B., et al. (2020). Comparative analysis of physicochemical properties and microbial composition in hightemperature Daqu with different colors. Front. Microbiol. 11:1-13. doi: 10.3389/ fmicb.2020.588117

Du, H., Wang, X. S., Zhang, Y. H., and Xu, Y. (2019). Exploring the impacts of raw materials and environments on the microbiota in Chinese Daqu starter. Int. J. Food Microbiol. 297, 32-40. doi: 10.1016/j.ijfoodmicro.2019.02.020

Fan, G. S., Fu, Z. L., Sun, B. G., Zhang, Y. H., Wang, X. L., Xia, Y. Q., et al. (2019). Roles of aging in the production of light-flavored Daqu. J. Biosci. Bioeng. 127, 309-317. doi: 10.1016/j.jbiosc.2018.08.005

\section{DATA AVAILABILITY STATEMENT}

The data presented in the study are deposited in the NCBI Sequence Read Archive (SRA) repository, accession number (PRJNA727444).

\section{AUTHOR CONTRIBUTIONS}

YZ designed and conducted the experimental work assisted by $\mathrm{XC}$, performed the data analysis, and wrote the first draft of the manuscript. YX contributed to the manuscript revision. B-ZH contributed to the supervision, manuscript revision, and overall support of this study. All authors read and approved the final version of the manuscript.

\section{FUNDING}

This work was supported by the National Natural Science Foundation of China (No. 31972975) and China Alcoholic Drinks Association (Migration of Wheat-originated Microorganisms in the Process of High-Temperature Daqu).

\section{SUPPLEMENTARY MATERIAL}

The Supplementary Material for this article can be found online at: https://www.frontiersin.org/articles/10.3389/fmicb. 2021.714726/full\#supplementary-material

Fan, G. S., Fu, Z. L., Teng, C., Liu, P. X., Wu, Q. H., Rahman, M. K. R., et al. (2020). Effects of aging on the quality of roasted sesame-like flavor Daqu. BMC Microbiol. 20:1-16. doi: 10.1186/s12866-020-01745-3

Fan, X. J., Jiao, X., Liu, J. G., Jia, M., Blanchard, C., and Zhou, Z. K. (2021). Characterizing the volatile compounds of different sorghum cultivars by both GC-MS and HS-GC-IMS. Food Res. Int. 140:109975. doi: 10.1016/j.foodres. 2020.109975

Fan, Y., Huang, X. N., Chen, J. Y., and Han, B. Z. (2020). Formation of a mixedspecies biofilm is a survival strategy for unculturable lactic acid bacteria and saccharomyces cerevisiae in Daqu, a Chinese traditional fermentation starter. Front. Microbiol. 11:1-13. doi: 10.3389/fmicb.2020.00138

Franco, M. F., Lori, G. A., Cendoya, M. G., Panelo, J. S., Alonso, M. P., Mirabella, N. E., et al. (2021). Using anthesis date as a covariate to accurately assessing type II resistance to Fusarium head blight in field-grown bread wheat. Crop Prot. 142:105504. doi: 10.1016/j.cropro.2020.105504

Gou, M., Wang, H. Z., Yuan, H. W., Zhang, W. X., Tang, Y. Q., and Kida, K. (2015). Characterization of the microbial community in three types of fermentation starters used for Chinese liquor production. J. Inst. Brew. 121, 620-627. doi: 10.1002/jib. 272

Guan, T. W., Lin, Y. J., Chen, K. B., Ou, M. Y., and Zhang, J. X. (2020). Physicochemical factors affecting microbiota dynamics during traditional solidstate fermentation of Chinese strong-flavor Baijiu. Front. Microbiol. 11:2090. doi: $10.3389 /$ fmicb. 2020.02090

He, Z. L., Fan, W. L., Xu, Y., He, S. G., and Liu, X. Y. (2019). Aroma profile of folium isatidis leaf as a raw material of making bingqu for chixiang aroma- and flavor-type Baijiu. ACS Symp. Ser. 1321, 263-275. doi: 10.1021/bk-2019-1321. ch016 
Huang, X. N., Fan, Y., Lu, T., Kang, J. M., Pang, X. N., Han, B. Z., et al. (2020). Composition and metabolic functions of the microbiome in fermented grain during light-flavor Baijiu fermentation. Microorg 8:1281. doi: 10.3390/ microorganisms8091281

Jin, G. Y., Zhu, Y., and Xu, Y. (2017). Mystery behind Chinese liquor fermentation. Trends Food Sci. Technol. 63, 18-28. doi: 10.1016/j.tifs.2017.02.016

Jin, Y., Li, D. Y., Ai, M., Tang, Q. X., Huang, J., Ding, X. F., et al. (2019). Correlation between volatile profiles and microbial communities: a metabonomic approach to study Jiang-flavor liquor Daqu. Food Res. Int. 121, 422-432. doi: 10.1016/j. foodres.2019.03.021

Knights, D., Kuczynski, J., Charlson, E. S., Zaneveld, J., Mozer, M. C., Collman, R. G., et al. (2011). Bayesian community-wide culture-independent microbial source tracking. Nat. Methods 8, 761-765. doi: 10.1038/nmeth. 1650

Langille, M. G. I., Zaneveld, J., Caporaso, J. G., McDonald, D., Knights, D., Reyes, J. A., et al. (2013). Predictive functional profiling of microbial communities using 16s rRNA marker gene sequences. Nat. Biotechnol. 31, 814-821. doi: $10.1038 /$ nbt. 2676

Leite, R. S. R., Alves-Prado, H. F., Cabral, H., Pagnocca, F. C., Gomes, E., and Da-Silva, R. (2008). Production and characteristics comparison of crude $\beta$-glucosidases produced by microorganisms Thermoascus aurantiacus e Aureobasidium pullulans in agricultural wastes. Enzyme Microb. Technol. 43, 391-395. doi: 10.1016/j.enzmictec.2008.07.006

Li, M. Q., Yang, R. W., Zhang, H., Wang, S. L., Chen, D., and Lin, S. Y. (2019). Development of a flavor fingerprint by HS-GC-IMS with PCA for volatile compounds of tricholoma matsutake singer. Food Chem. 29, 32-39. doi: 10. 1016/j.foodchem.2019.03.124

Li, P., Lin, W. F., Liu, X., Wang, X. W., Gan, X., Luo, L. X., et al. (2017). Effect of bioaugmented inoculation on microbiota dynamics during solid-state fermentation of Daqu starter using autochthonous of bacillus, Pediococcus, Wickerhamomyces and Saccharomycopsis. Food Microbiol. 61, 83-92. doi: 10. 1016/j.fm.2016.09.004

Li, R. Y., Zheng, X. W., Zhang, X., Yan, Z., Wang, X. Y., and Han, B. Z. (2018). Characterization of bacteria and yeasts isolated from traditional fermentation starter (Fen-Daqu) through a ${ }^{1} \mathrm{H}$ NMR-based metabolomics approach. Food Microbiol. 76, 11-20. doi: 10.1016/j.fm.2018.03.015

Li, W. W., Fan, G. S., Fu, Z. L., Wang, W. H., Xu, Y. Q., Teng, C., et al. (2020). Effects of fortification of Daqu with various yeasts on microbial community structure and flavor metabolism. Food Res. Int. 129:108837. doi: 10.1016/j.foodres.2019. 108837

Liu, C. C., Feng, S. B., Wu, Q., Huang, H. Q., Chen, Z. X., Li, S. W., et al. (2019). Raw material regulates flavor formation via driving microbiota in Chinese liquor fermentation. Front. Microbiol. 10:1-13. doi: 10.3389/fmicb.2019.01520

Márquez-Sillero, I., Aguilera-Herrador, E., Cárdenas, S., and Valcárcel, M. (2011). Ion-mobility spectrometry for environmental analysis. TrAC-Trends Anal. Chem. 30, 677-690. doi: 10.1016/j.trac.2010.12.007

Pang, X. N., Huang, X. N., Chen, J. Y., Yu, H. X., Wang, X. Y., and Han, B. Z. (2020). Exploring the diversity and role of microbiota during material pretreatment of light-flavor Baijiu. Food Microbiol. 91:103514. doi: 10.1016/j.fm.2020.103514

Pradhan, P., and Tamang, J. P. (2019). Phenotypic and genotypic identification of bacteria isolated from traditionally prepared dry starters of the Eastern Himalayas. Front. Microbiol. 10:2526. doi: 10.3389/fmicb.2019.02526

Siddiquee, S. (2018). Recent advancements on the role of biologically active secondary metabolites from Aspergillus. N. Futur. Dev. Microb. Biotechnol. Bioeng. 69-94. doi: 10.1016/B978-0-444-63501-3.00004-1

Stevens, D., Cornmell, R., Taylor, D., Grimshaw, S. G., Riazanskaia, S., Arnold, D. S., et al. (2015). Spatial variations in the microbial community structure and diversity of the human foot is associated with the production of odorous volatiles. FEMS Microbiol. Ecol. 91, 1-11. doi: 10.1093/femsec/fiu018

Wang, Y. R., Cai, W. C., Wang, W. P., Shu, N., Zhang, Z. D., Hou, Q. C., et al. (2021). Analysis of microbial diversity and functional differences in different types of high-temperature Daqu. Food Sci. Nutr. 9, 1003-1016. doi: 10.1002/ fsn 3.2068

Wei, J. L., Du, H., Zhang, H. X., Nie, Y., and Xu, Y. (2021). Mannitol and erythritol reduce the ethanol yield during Chinese Baijiu production. Int. J. Food Microbiol. 337:108933. doi: 10.1016/j.ijfoodmicro.2020.108933

Wu, X. H., Zheng, X. W., Han, B. Z., Vervoort, J., and Robert Nout, M. J. (2009). Characterization of Chinese liquor starter, "Daqu" by flavor type with ${ }^{1} \mathrm{H}$ NMRbased nontargeted analysis. J. Agric. Food Chem. 57, 11354-11359. doi: 10.1021/ jf902881p

Xu, Y., Wang, D., Fan, W. L., Mu, X. Q., and Chen, J. (2010). Traditional Chinese biotechnology. Adv. Biochem. Eng. Biotechnol. 122, 189-233. doi: 10.1007/10 2008_36

Yan, Z. K., Meng, Q. Y., Wang, X. Z., and Du, J. (2016). Analysis on physicochemical indexes and cultivation technology of traditional Xifeng Daqu sensory evaluation. Liquor Making 43, 51-55.

Yang, F., Liu, Y., Wang, B., Song, H. L., and Zou, T. T. (2020). Screening of the volatile compounds in fresh and thermally treated watermelon juice via headspace-gas chromatography-ion mobility spectrometry and comprehensive two-dimensional gas chromatography-olfactory-mass spectrometry analysis. LWT 137:110478. doi: 10.1016/j.lwt.2020.110478

Yu, R. L., Li, M. Z., Wang, Y., Bai, X. L., Chen, J. W., Li, X. N., et al. (2021). Chemical investigation of a co-culture of aspergillus fumigatus $\mathrm{d}$ and fusarium oxysporum r1. Rec. Nat. Prod. 15, 130-135. doi: 10.25135/rnp.199.20.07.1728

Zhang, X. Y., Wang, C. C., Wang, L. L., Chen, S., and Xu, Y. (2020). Optimization and validation of a head space solid-phase microextractionarrow gas chromatography-mass spectrometry method using central composite design for determination of aroma compounds in Chinese liquor (Baijiu). J. Chromatogr. A 1610:460584. doi: 10.1016/j.chroma.2019.460584

Zheng, X. W., and Han, B. Z. (2016). Baijiu (白酒), Chinese liquor: History, classification and manufacture. J. Ethn. Foods 3, 19-25. doi: 10.1016/j.jef.2016. 03.001

Zheng, X. W., Tabrizi, M. R., Robert Nout, M. J., and Han, B. Z. (2011). Daqua traditional Chinese liquor fermentation starter. J. Inst. Brew. 117, 82-90. doi: 10.1002/j.2050-0416.2011.tb00447.x

Zheng, X. W., Yan, Z., Han, B. Z., Zwietering, M. H., Samson, R. A., Boekhout, T., et al. (2012). Complex microbiota of a Chinese "Fen" liquor fermentation starter (Fen-Daqu), revealed by culture-dependent and culture-independent methods. Food Microbiol. 31, 293-300. doi: 10.1016/j.fm.2012.03.008

Conflict of Interest: YS, WC, and XW were all employed by Sichuan Langjiu Co., Ltd.

The remaining authors declare that the research was conducted in the absence of any commercial or financial relationships that could be construed as a potential conflict of interest.

Copyright (c) 2021 Zhang, Shen, Cheng, Wang, Xue, Chen and Han. This is an open-access article distributed under the terms of the Creative Commons Attribution License (CC BY). The use, distribution or reproduction in other forums is permitted, provided the original author(s) and the copyright owner(s) are credited and that the original publication in this journal is cited, in accordance with accepted academic practice. No use, distribution or reproduction is permitted which does not comply with these terms. 